\title{
Prevalence of Pneumonia in Children'S Under Five Years in Al Sabbah Hospital Juba - South Sudan
}

\section{Simon D Nyicar}

Upper Nile University

\section{David A. Amum}

Upper Nile University

Philip E. Kutjok ( $\nabla$ pkutjok@yahoo.com )

Upper Nile University

\section{Pakjwok J. Aban}

Upper Nile University

Opwonyi N. Awin

Upper Nile University

Tuna Z. Nyiboy

Public Health Laboratory, Juba- South Sudan

Angelo A. Kol

Upper Nile University

\section{Athieng M. Angong}

Upper Nile University

Lam M. Abraham

Upper Nile University

Mandela M. Jiek

Upper Nile University

Rahiel J. khamis

Upper Nile University

\section{Research}

Keywords: Prevalence, Pneumonia, under - five year, Al - Sabah Children Hospital, South Sudan

Posted Date: December 15th, 2021

DOI: https://doi.org/10.21203/rs.3.rs-1068110/v1

License: (9) (1) This work is licensed under a Creative Commons Attribution 4.0 International License.

Read Full License 


\section{Abstract}

Background: Each year, pneumonia affects about 450 million people globally; it is a major cause of death among all age groups resulting in 4 million deaths ( $7 \%$ of the world's total death) yearly.

Pneumonia is defined as the presence of fever, acute respiratory symptoms, or both, plus evidence of parenchymal infiltration chest radiography.

Although, in the past decade the incidence of the disease was reported to have declined by half percent, that alone was considered to be the slowest decline in comparison to other diseases

The objective of the study is to determine the prevalence of pneumonia in Al Sabah Children Hospital and to assess the risk factor associated.

Method: A cross-sectional study design was used to determine the prevalence of pneumonia in children under-five years in Al Sabah hospital, Juba South Sudan. The study ran from $18^{\text {th }}$ September to $5^{\text {th }}$ of October 2021. 241 participants were randomly selected for the study and primary data were obtained through questionnaires.

Results: The prevalence of pneumonia in children under-five years was found to be $22.4 .7 \%$.

The incidence is highest in males $23(57.5 \%)$ compared to females17 (42.5\%). The ethnicity of the patients from Dinka (37.5\%) and Bari (30\%), Shilluk (7.5\%), Mundari (2.5\%), Madi (5\%) Nuer (7.5\%), Murli $(5 \%)$, Lolobo $(2.5 \%)$ and latoka $(2.5 \%)$ and the leading ethnicity with high morbidity of pneumonia was found to be in Dinka.

Conclusion: The prevalence of pneumonia in children under-five years in Al Sabah hospital is high compared to the previous research done by the students of Upper Nile University at the University of Juba in 2019 with the prevalence of $21 \%$

\section{Background}

Pneumonia is an in־ammation of the Lungs' parenchymal structure ${ }^{1}$. It is also defined as an inflammatory Condition of lung primarily affecting small air sacs known as alveoli ${ }^{2}$. Each year, pneumonia affects about 450 million people globally; it is a major cause of death among all age groups resulting in 4 million deaths ( $7 \%$ of the world's total death) yearly. Rates are greatest in children less than five, and adults less than 75 years ${ }^{3}$

Pneumonia kills more children than any other infectious disease, claiming the lives of over 800,000 children under five every year, or around 2,200 every day. This includes over 153,000 newborns ${ }^{4}$. Almost all of these deaths are preventable. Globally, there are over 1,400 cases of pneumonia per 100,000 children, or 1 case per 71 children every year, with the greatest incidence occurring in South Asia $(2,500$ cases per 100,000 children) and West and Central Africa (1,620 cases per 100,000 children). ${ }^{5}$ 
In South Sudan, pneumonia is one of the top diseases causing morbidity and mortality among young children. It was reported that the disease was responsible for one-fifth of deaths of children under five years in $2018^{6}$

A report by the UN children's agency UNICEF and charity groups Save the Children and Every Breath Counts Coalition, said pneumonia claimed 7,640 lives of children under the age of five in 2018 in the country, causing 20 percent of under-five deaths due to poverty and lack of access to health services. ${ }^{6}$

Children immune compromised by malnutrition, and those living in areas with high levels of air pollution and unsafe water, are at far greater risk, the aid agencies said in a joint statement. ${ }^{6}$

Clinically, pneumonia is defined as the presence of fever, acute respiratory symptoms, or both, plus evidence of parenchymal infiltration chest radiography. ${ }^{7}$ In the worst situation, it can affect the lung where the alveoli are filled with pus and fluid, which makes breathing painful and limits oxygen intake. ${ }^{7}$ There are a large number of microorganisms that can cause pneumonia, such as bacteria which is the most common cause of community acquired pneumonia, with streptococcus pneumonia isolated in nearly $50 \%$, homophiles influenza in $20 \%$, chlamydophila pneumonia in $13 \%$ Mycoplasma pneumonia in $3 \%$ of cases and viruses which account for about one third of pneumonia cases in adults, and in children for about $15 \%$ of them. ${ }^{8}$ Commonly implicated agents include rhinoviruses, corona viruses, influenza virus respiratory syncytial virus, and adenovirus and Para influenza. Fungal pneumonia is uncommon but occurs more commonly in individual with weakened immune ${ }^{8}$

\subsection{Scope of Problem}

Pneumonia is responsible for the deaths of more than 800,000 young children worldwide each year, according to the United Nations Children's Fund (UNICEF) ${ }^{4}$. These deaths occur almost exclusively in children with underlying conditions, such as chronic lung disease of prematurity, congenital heart disease, and immunosuppressant. Pneumonia accounts for $15 \%$ of all deaths of children under 5 years old, killing 808694 children in 2017. Globally, there are over 1,400 cases of pneumonia per 100,000 children, or 1 case per 71 children every year, with the greatest incidence occurring in South Asia (2,500 cases per 100,000 children) and West and Central Africa (1,620 cases per 100,000 children).

The disease is one of the leading killers of children in South Sudan. Causing $20 \%$ of fewer than five deaths in 2018 due to poverty, and lack of access to health. ${ }^{9}$

\section{2 knowledge Gap}

Health facilities in South Sudan are overwhelmed with pneumonia cases with more than 7,000 deaths in 2018. Pneumonia in South Sudan complicated with other childhood diseases like anemia and malaria, and a lack of access to health services makes things more dangerous

\subsection{Problem Statement}


The prevalence of pneumonia in children under five is assumed increasing. However, there is no clear data supporting this increment in South Sudan.

\subsection{Research Question}

What is the prevalence of pneumonia in children under five years in Al Sabah Hospital?

\subsection{Objectives}

\subsubsection{Overall Objective}

The overall objectives of this study is to determine the environmental, social-economic and nutritional risk factors associated with children less than five years of age hospitalized with pneumonia in Al Sabah Hospital .

\subsubsection{Specific Objectives}

- To identify the major risk factors of pneumonia in children under-five years Al Sabah Hospital.

- To determine the prevalence of pneumonia in children under five years in Al Sabah Hospital.

- To Reduce the prevalence of Pneumonia in children under five years in Al Sabah

Hospital by providing evidence based information's to policy makers that would help them take correct decisions

\subsection{Public Health Significance}

In the world where numerous etiological and risk factors had already been identified, leading to the availability of preventive and curative measures, pneumonia still remains a major challenge to child health and survival in South Sudan ${ }^{9}$. Hence the significance of this research aimed at various levels including the community and the health policy makers are as follows:

- The research is also of profound value in planning, adopting and promoting policies that prevent and reduce morbidity and mortality by increasing the understanding to the need for vaccine coverage, antibiotics and other medication to all parts of the country.

- Lack of awareness and education of the mothers may adversely have an impact on the outcome of the illness which may be amenable to public health intervention.

- Educated mothers recognize the signs and symptoms of pneumonia early and so access health care earlier and so their children have a better outcome than others.

- A study conducted in Nigeria reveals that poor parental educational status is significantly associated with ARI.

- Study has found significant association of prelacteal feeding with childhood pneumonia.

- Age at onset of complementary feeding may also be associated with pneumonia occurrence ${ }^{9}$. Occurrence is $21.3 \%$ when feeding was started at 4 months, $13.7 \%$ at six months and $30.7 \%$ beyond 
six months of age. ${ }^{9}$ Our study too shows statistical association of complementary feeding in time with pneumonia occurrence.

- Immunization of children with two doses of measles vaccine through routine immunization, immunization with three doses of pentavalent vaccine and also booster doses of DPT vaccine during childhood can go a long way for prevention of pneumonia occurrence.

- Reductions in occurrence of pneumonia have been observed in completely immunized child as according to age.

In this chapter, we have focused on hypothesis, research question, objective and public health significance of pneumonia. In the next chapter therefore, we shall discuss about the mortality rate, classifications, risk factors associated with it and managements of pneumonia.

\section{Research Methodology}

\subsection{Study Design}

This was a descriptive cross-sectional study. Although cause and effect cannot be established by this study design, we chose it because it can determine the prevalence, less expensive and take short duration.

\subsection{Site of the Study}

The study was conducted in Al - Sabah Children Hospital in Central Equatoria State, Juba South Sudan. The hospital is located at Hai Cinema next to Jerusalem House.

It is the main children hospital in Juba, with an outpatient capacity of 250_300 per day and admits about $25 \_40$ patients per day and a capacity of seven wards.

The hospital is served by 200 staff both classified (doctors, nurses and lab technical and public health officers) and unclassified (cleaners etc).

\subsection{Population of the Study}

The study is done in Al - Sabah Children Hospital population of children under the age of five years from January to June 2021.

\subsection{Sample Size}

The Sample size was 241 .

\subsection{Variables}

The study of mortality rate and risk factors of pneumonia was done. Patient and Parents or maternal related risk factors, Environment related risk factors, Malnutrition and Vitamin A deficiency, Low birth weight, Air pollution, viral infections and level of management were also analysed. 


\subsection{Source of Data:}

Al - Sabbah Children Hospital will be the source of data collection for this study.

\subsection{Collection of Data:}

The activity designed to collect data took 18 days from 18 of September to 5 th of October 2021. A Questionnaire was also developed for interviewing the different care givers in population of children under five years in addition; the study also applied focus group discussion and observation as some of scientific methodologies for data collection.

\subsection{Data Management and Statistical Analysis:}

The collected data will be first reassessed for compatibility and accomplishment, and so will be arranged, presented or displayed in the form of graphs, charts and frequency tables using Microsoft Excel and word 2013.

\subsection{Limitations}

In the search for up to date information about the prevalence of pneumonia in Central Equatoria State, the lack of medical data in the country has forced us to entirely depend on primary data. The burden doesn't start and end only with the cost for the data collection techniques which included:

- Printing of the questionnaires.

- Transport to and from the hospital.

- Infinity in terms of language barrier.

- Time consumption.

- Has a potential for response bias (e.g. level of literacy)

- Can't establish whether an exposure will be followed by outcome (temporality)

- Not cooperative parents

\section{Results}

\subsection{Analysis of Results}

Our sample size comprises of 241 under-five years children of which male was $116(48 \%)$ and the remaining is female which 152 is (52\%). 
Table 1.1

Distribution of the Study Sample by Cause of Admission

\begin{tabular}{|lll|}
\hline Cause of admission & Frequency & Relative frequency \\
\hline pneumonia & 57 & $23.6 \%$ \\
\hline Pneumonia with malaria & 93 & $38.5 \%$ \\
\hline Pneumonia with sepsis & 13 & $5.3 \%$ \\
\hline Pneumonia with asthma & 2 & $1.1 \%$ \\
\hline Pneumonia with meningitis & 7 & $3 \%$ \\
\hline Pneumonia with dehydration & 13 & $5.3 \%$ \\
\hline Death & 56 & $23.2 \%$ \\
\hline Total & 241 & $100 \%$ \\
\hline
\end{tabular}

\section{Demographic Characteristic of the Respondents}

The results in figure 3.1 showed that the risk of pneumonia was higher among Dinka ethnicity under-five children when compared to under-five children from other ethnicities.

The results in figure 3.2 showed that the risk of pneumonia was higher among under-five children residing in urban area 14 children, while lower among those under-five children who residing in rural area 7 children.

The results in figure 3.3 showed that the risk of pneumonia was higher among female under-five children 13 children than among male under-five children 8 children.

Surprisingly, the results in figure 3.4 showed that the risk of pneumonia was higher among under-five children who were born at hospital 17 children, while lower among those under-five children who were born at home 4 children. 
Table 3.2

Environmental Covariates

\begin{tabular}{|llcccc|}
\hline Variable & B & Sig. & Exp(B) & \multicolumn{2}{c|}{$95.0 \%$ Cl for Exp(B) } \\
\cline { 5 - 7 } & & & & Lower & Upper \\
\hline Type o family & 0.685 & 0.294 & 1.983 & .552 & 7.127 \\
\hline No. family members & -0.033 & 0.943 & 0.967 & .387 & 2.418 \\
\hline Type of home & 0.380 & 0.540 & 1.463 & .433 & 4.940 \\
\hline Overcrowding & -0.525 & 0.289 & 0.591 & .224 & 1.561 \\
\hline Method of cooking & -0.678 & 0.158 & 0.508 & .198 & 1.302 \\
\hline Baby care taker while her mother is cooking & 0.126 & 0.723 & 1.135 & .565 & 2.279 \\
\hline
\end{tabular}

Table 3.2 showed that type of family was positively and insignificantly linked with under-five children at $95 \%$ confidence level.

Type of home was positively and insignificantly allied with under-five children at the $95 \%$ confidence level. As expected, the risk of pneumonia among under-five children who were living in local building was about 1.463 times higher than those who lived in modern building.

Overcrowding was negatively and insignificantly associated with under-five children at $95 \%$ confidence level.

Method of cooking was negatively and insignificantly associated with under-five children at $95 \%$ confidence level..

Baby care taker was positively and insignificantly associated with under-five children at the $95 \%$ confidence level.

Table 3.3

Past History Related Covariates with Severe Pneumonia

\begin{tabular}{|llllll|}
\hline Variable & B & Sig. & $\operatorname{Exp}(\mathrm{B})$ & \multicolumn{2}{c|}{$\mathbf{9 5 . 0 \%} \mathrm{Cl}$ for $\operatorname{Exp}(\mathrm{B})$} \\
\cline { 5 - 7 } & & & & Lower & Upper \\
\hline Exclusive breastfeeding & 1.067 & 0.143 & 2.907 & .696 & 12.137 \\
\hline Vitamin A supplementations & 1.601 & 0.004 & 4.959 & 1.655 & 14.863 \\
\hline Hospitalization & -1.749 & 0.041 & 0.174 & .033 & .928 \\
\hline Home treatment & 2.018 & 0.021 & 7.526 & 1.353 & 41.852 \\
\hline
\end{tabular}

From Table 3.3 it was clear that exclusive breastfeeding was positively and insignificantly associated with under-five children at $95 \%$ confidence level. Vitamin A supplementation was positively and 
significantly linked with under-five children at $95 \%$ confidence level

Hospitalization was negatively and significantly allied with under-five children at the $95 \%$ confidence level.

Home treatment tried was positively and significantly associated with under-five children at $95 \%$ confidence level.

Table 3.4

Socio-Demographic and Birth Related Covariates

\begin{tabular}{|llllll|}
\hline & B & Sig. & Exp(B) & \multicolumn{2}{c|}{$95.0 \%$ Cl for Exp(B) } \\
\cline { 5 - 7 } & & & & Lower & Upper \\
\hline Born premature & -0.428 & 0.428 & 0.652 & .226 & 1.879 \\
\hline Born low birth weight & -0.468 & 0.447 & 0.626 & .188 & 2.089 \\
\hline Mother education & 0.199 & 0.633 & 1.220 & .539 & 2.762 \\
\hline Father education & -0.302 & 0.369 & 0.740 & .383 & 1.428 \\
\hline Mother occupation & -1.456 & 0.031 & 0.233 & .062 & .873 \\
\hline Father occupation & -0.146 & 0.833 & 0.864 & .222 & 3.358 \\
\hline Poor ventilation & 0.249 & 0.701 & 1.283 & .359 & 4.586 \\
\hline Contact with TB & 1.606 & 0.035 & 4.983 & 1.120 & 22.163 \\
\hline Smoking at home & -1.187 & 0.118 & 0.305 & .069 & 1.351 \\
\hline Drinking water Source & -0.290 & 0.663 & 0.749 & .204 & 2.751 \\
\hline Open defecation & 0.167 & 0.828 & 1.182 & .260 & 5.367 \\
\hline
\end{tabular}

The results in Table 3.4 showed that born premature was negatively and insignificantly associated with under-five children at the $95 \%$ confidence level.

Mother's education was positively and insignificantly associated with under-five children at $95 \%$ confidence level

Father's educational status was negatively and insignificantly linked with under-five children.

Mother's occupation was negatively and significantly associated with under-five children at the $95 \%$ confidence level.

Father's occupation was negatively and significantly associated with under-five children.

Poor ventilation was positively and insignificantly related to under-five children. 
Smoking at home was negatively and insignificantly connected to under-five children at $95 \%$ confidence level.

Drinking water Source was negatively and insignificantly associated to under-five children

Open defecation was positively and insignificantly associated with pneumonia in under-five children at the $95 \%$ confidence level.

\section{Challenges of this Study:}

1. Maternal and or caretakers have low Education level. Most if not all are illiterate. Hence information filled may not be correct. Information such as the Age and Detail of child birth history.

2. Poor hospital records

3. Refusal to answer questions

4. failure of the parents to cooperate

\subsection{Findings from Other Scientific Methodologies 3.2.1 Focus Group Discussion}

In order to obtain more information to strengthen our research, we also opted to FGD as one of the scientific methodologies. The aim was to discuss the risk factors associated with pneumonia, the current available intervention measure and to get idea on what can be done to prevent pneumonia in Al Sabbah Children Hospital.

According to 40 cases of children with pneumonia, the main risk factor was cold weather and rainy season. Other risk factors that were identified include; smoking, dust, and Air pollution. One of the participants highlighted that when a child Ss not breast fed exclusively for more than six months and not vaccinated it can become sick with pneumonia and other related diseases.

Other women said that they were not aware of any intervention measures put in place to prevent and control pneumonia in Al-Sabah children hospital. However, according to Christina one of the mothers, who brought her child for treatment in AI Sabah hospital, noted that the time she brought her child, the child was screened for pneumonia. She also added that a seminar for managing pneumonia at home was conducted. Most participants believed that creating public awareness about risk factor associated with Pneumonia, the prevalence of pneumonia would be reduced.

A pediatrician categorized the risk factors such as the air pollution, coldness, infected person and the dust. However, she also added that the following risk factors play a significant role in predisposing to pneumonia particularly among the children, getting coldness and smoking history of spouses. She noted that exposing a child to cold environment is very potential risk factor. 


\subsubsection{Interview}

We interviewed 15 participants who comprised of 3 care givers, 5 from department of Nutrition and other 7 from staff within Al-Sabah Children Hospital. The aim of the interview was to access the level of awareness, the associated risk factors and intervention measures that had been or can be put in place to prevent and control the prevalence of pneumonia in Al-Sabah Children Hospital.

Generally the level of awareness was moderate among the participants 15 of participant ( 5 care givers, 7 staffs and 5 nutrition officers) said they had heard about pneumonia. And these, only 5 participants ( 1 care giver, 4 staff and 2 nutrition officers) were capable of giving clear cut explanation about pneumonia.

The following measures were suggested by subjects in order to prevent and control the prevalence of pneumonia,

- The government must provide drugs for pneumonia in different health centers

- Improvement of health care facility across the country,

- Pneumonia awareness programs should be carried out in communities

\subsubsection{Observation}

The level of awareness about pneumonia among mothers in Al-Sabah children hospital varies. It appeared to be highest in children of educated and employed mothers. However, mothers who are not educated had the lowest level of awareness.

From our observation, no intervention measures had been put in place to prevent and control pneumonia. However, mothers were eager to learn more about the prevention and control of pneumonia. We also noted that there were increased new cases. This is from the statistics. Possibly it may be because of changes in the season.

\section{Discussion}

The finding shows that the prevalence of pneumonia in children under-five years in Al Sabah hospital is $22.4 \%$. This is higher compared to the previous research done by the students of Upper Nile University and Juba University in 2019 with the prevalence of $21 \%$. The increase in prevalence can be best explained by taking into consideration the discussion of the commonly associated risk factors in context with the country.

This study shown earlier in chapter four indicates that the incidence of pneumonia is highest in children under five years. This shows that children less than one year of age are at more risk to pneumonia compared to the other age groups of less than five years. This can be linked to the combination of multiple factors including nonexclusive breastfeeding, the behavior of the children as they begin to move, 
getting exposed to different environmental factors and more importantly is that, most of these children might have not yet completed the recommended childhood vaccines at that age group.

Of total 40 pneumonia cases, 23 were males that make $57.5 \% \%$ and 17 were females making $42.5 \%$. Moreover, this study confirms with the other studies that males were more susceptible to contract the disease compared to the females. This fact is more within the age group under five years.

In accordance with this study, the ethnicity of the patients from Dinka (37.5\%) and Bari (30\%), Shilluk (7.5\%), Mundari (2.5\%), Madi (5\%) Nuer (7.5\%), Murli (5\%), Lolobo $(2.5 \%)$ and latoka (2.5\%) are the leading ethnicities with high morbidity of pneumonia the highest was found in Dinka. This can be related to the factors such as population density and socioeconomic status. Dinka is known to be one of the highest population in Juba city. On the other hand, Lolobo, Mundari and Latoko are one of few ethnic groups in Juba city .

The data showed that premature birth was negatively and insignificantly associated with under-five children at the $95 \%$ confidence level. The risk of pneumonia was 0.652 times lower among under-five children who have not been born premature than those under-five children who have born premature. Similarly, low birth weight was negatively and not significantly connected with under-five children. The risk of pneumonia was 0.626 times lower among under-five children who had been born with lower weight than those under-five children who had been born with higher birth weight.

Mother education was positively and insignificantly associated with under-five children at $95 \%$ confidence level. As expected, the risk of pneumonia among under-five children whose mothers were illiterate was about 1.220 times higher than those under-five children whose mothers with primary education and below. Father education was negatively and insignificantly linked with under-five children. The risk of pneumonia among under-five children whose fathers had primary education and above was around 0.740 times lower than among those under-five children whose fathers were illiterate.

Mother's occupation was negatively and significantly associated with under-five children at the 95\% confidence level. As expected, the risk of pneumonia among under-five children whose mothers were working was about 0.233 times lower as compared to those under-five children whose mothers were not working. Similarly, father occupation was negatively and significantly associated with under-five children. The risk of pneumonia among under-five children whose fathers were working was about 0.864 times lower than those under-five children whose fathers were not working.

Poor ventilation was positively and insignificantly related to under-five children. The risk of pneumonia was about 1.283 times higher in households with poor ventilation. Besides that, contact with TB was positively and significantly associated with pneumonia in under-five children at the $95 \%$ confidence level.

Smoking at home was negatively and insignificantly connected to under-five children at $95 \%$ confidence level. The risk of pneumonia among under-five children in homes with no smoking was around 0.305 times lower than those in homes with smoking. 
Drinking water source was negatively and insignificantly associated with under-five children. The risk of pneumonia was about 0.749 times lower among under-five children living in households whose source of drinking water is tap water than those under-five children living in households their source of drinking water is underground water. Open defecation was positively and insignificantly associated with pneumonia in under-five children at the $95 \%$ confidence level.

Type of family was positively and insignificantly linked with under-five children at $95 \%$ confidence level. The risk of pneumonia for under-five children who living in joint family was about 1.983 times higher compared with under-five children who were living in nuclear family.

Number of family members was negatively and insignificantly associated with under-five children at $95 \%$ confidence level. As expected, the risk of pneumonia for under-five children who were living in family with members up to 5 persons was about 0.967 times lower as compared to those children who were living in family their members more than 5 persons.

Type of home was positively and insignificantly allied with under-five children at the $95 \%$ confidence level. As expected, the risk of pneumonia among under-five children living in local building was about 1.463 times higher than those in modern building.

Overcrowding was negatively and insignificantly associated with under-five children at $95 \%$ confidence level. As expected, the risk of pneumonia for under-five children living in uncrowded houses was about 0.591 times lower than those under-five children living in crowded houses.

Method of cooking was negatively and insignificantly associated with under-five children at $95 \%$ confidence level. As expected, the risk of pneumonia for under-five children whose mothers were cooking with gas was about 0.508 times lower than those under-five children whose mothers were cooking with firewood and charcoal.

\subsection{Recommendations}

The recommendations cover Health Information Education and Communication about preventive measures that target the Government, Health workers and Development partners. It also targets the entire population including women, men, youth and community leaders. There should be a development of community health programs that aim at promoting health education by creating awareness about the causes, risk factors and prevention of the disease via the radio, television, or health campaigns in the schools and churches and other social institutions:

Educate parents to understand that local herbs, home prescribed medications can prevent a child from getting appropriate treatment at the right time which can lead to further complications of the disease.

- Train health workers on GOBIFFF principles (Growth monitoring, oral rehydration, breastfeeding, Immunization, female education, family planning and food supplement) 
- Provision of medical supplies and improve laboratory services

- Educate the parents and caretakers about child health care

- Training parents about the importance of good nutrition for both the child and mother in prevention of malnutrition, low birth weight and associated disease that can predispose to pneumonia.

- Educate the caretakers on the importance of the completion of all the recommended vaccines for their children.

- Mobilizing the parents on the importance of urgent medical intervention in preventing the complications of the disease.

- Provision of clean and safe drinking water especially in displacement camps

- Provision of clean form of energy such as electricity and solar energy

- Provision of the required medications for the treatment of pneumonia in all the health facilities.

- Provision of medications for other diseases that can predispose a child to pneumonia.

- Continuous data collection, monitoring and evaluation of the disease progress.

- Educating the community to build better ventilated houses

- Educating the community about the dangers of smoking

- Advocating the government to develop a policy of managing smoking

- Advocating the development partners such UNICEF to support programs related to child health care.

- Allocate budget for research and encourage young researchers to look more into disease dimensions

\section{Conclusion}

The prevalence of pneumonia in children under-five years in Al Sabah hospital is high compared to the previous research done by the students of Upper Nile and Juba University in 2019 with the prevalence was $21 \%$.

In light of this, there should be a development of community health programs that aim at promoting health education by creating awareness about the causes, risk factors and prevention of the disease via the radio, television, or health campaigns in the schools and churches and other social institutions and a further studies to be conducted at states level

\section{Abbreviations}

UN UNITED NATIONS

UNICEF UNITED NATIONS INTERNATIONAL CHILDREN'S FUND

ARI ACUTE RESPIRATORY FAILURE

DPT DIPHTHERIA PERTUSSIS TETANUS

Page $14 / 20$ 
CAP COMMUNITY ACQUIRED PNEUMONIA

HAP HOSPITAL ACQUIRED PNEUMONIA

VAP VENTILATOR -ASSOCIATED PNEUMONIA

RSV RESPIRATORY SYNCYTIAL VIRUS

SARS-COV-2 SEVERE ACUTE RESPIRATORY - CORONA VIRUS - 2

COVID-19 CORONA VIRUS DISEASE 2019

COPD CORONARY OBSTRUCTED PULMONARY DISEASE

CMV CYTOMEGALOVIRUS

AIDS ACQUIRE IMMUNODEFICIENCY SYNDROME

NPI NATIONAL PNEUMONIA IMMMUNAZITION

TB TUBERCOLOSIS

HIV HUMAN IMMUNODEFICIENCY VIRUS

LDH LACTATE DEHYDROGENASE

\section{Declarations}

\section{Ethics approval and consent to participate:}

Permission to carry out the study was granted by the ethical committee, faculty of medicine in Upper Nile University and Al - Sabah Children Hospital.

\section{Consent for publication}

Informed consent were obtained for the questioners from the participants prior to data collection. Anybody who declined their data are not collected and they were excluded.

Patients' information that were obtained, were kept confidential and an effort was made to maintain the privacy and autonomy of the participants by encrypting all the information through the process of data analysis.

\section{Availability of data and material}

Datasets used and/or analyzed during the current study are available from the corresponding author on reasonable request. 


\section{Funding:}

Not Applicable

\section{Competing interests}

The authors declare that they have no competing interests

\section{Authors' contributions}

Ak, AA, LA, MJ and RK were very helpful in data collection for this research. PK, PA, OW and TZ were a major contributors to writing the manuscript. SN gave a final review and approval to the manuscript and finally DA analyzed and interpreted the patient data statistically.

All authors read and approved the final manuscript.

\section{Acknowledgements}

We hereby would like to thank everyone who supported us directly or indirectly during the course of our research.

We would also like to thank the staff members of Department of Community Medicine, Upper Nile University for their counsel especially in the beginning of our research project.

Similarly, our warm gratitude to the administration of Al - Sabbah Hospital for their cooperation and for granting us permission to conduct the study.

All the parents who participated in this study by accepting to provide for us all the information we would ever need for the success of the study.

And finally, to all the members of the research team for exhausting their precious time, energy and resources all the way from day one to the time of approval whether through searching, collecting, organizing or interpreting the data and Special thanks to Prof. Tom Yolong for the language review.

\section{Authors' information}

${ }^{1}$ Faculty of Medicine, Department of Microbiology, Upper Nile University, South Sudan. ${ }^{2}$ Faculty of Medicine, Department of Medical statistics and population studies, Upper Nile University, South Sudan. ${ }^{3}$ Faculty of Medicine, Department of Community Medicine, Upper Nile University, South Sudan. ${ }^{4}$ Faculty of Medicine, Department of Biochemistry, Upper Nile University, South Sudan. ${ }^{5}$ Faculty of Medicine, Department of Microbiology, Upper Nile University, South Sudan. ${ }^{7}$ Public Health Laboratory, Juba- South Sudan. ${ }^{7}$ School of Medicine, Upper Nile University, South Sudan

\section{References}


1. Prevalence of pneumonia and its associated factors among under-five children in East Africa: a systematic review and meta-analysis. Biruk Beletew, Melaku Bimerew, Ayelign Mengesha,. 27 May 2020, BMC Pediatrics, p. 1.

2. Respiratory disease and its managment. mclukie A, ed . new york : s.n., 2009, p. 51.

3. Viral pneumonia. ruuskanan o, lahti e, jenning lc murduch dr. s.I. : 2011, april 2011, p. 377.

4. PNEUMONIA. WHO. 2019, p. 1.

5. WHO. who maternal child epidemology estimation. unicef data. s.I. : 2012, 2018. p. 2, covid 19 latest information.

6. South Sudan lost 7,640. xinhua. s.I. : 2019, 2018, p. 6.

7. An analysis of the prevalence of pneumonia for children under 12 year old in tawau hospital, malaysia. suliadi f . sufahni, sit $\mathbf{n}$.mohd razail. mohammad f.mormin ,azme kamis. s.I. : 2012, p. 2.

8. Radiological imaging in peumonia; recent innovation. current opinion in pulmonary medicine. sharma s, maycher b, eschunun g. s.I. : 2020, 2007.

9. Fighting for breath in south sudan. save the children, unicef, every breath counts. s.I. : 2020, 2017, p. 2.

10. Pneumonia. Bernadeta Dadonaite, Max rosar. s.I. : 2019, 2019, p. 3.

11. Kumar P, Clarks C. clinical Medicine. Seventh edition. Iondon : s.n., 2009. p. 2715.

12. A, Mohammed. Nelson pediatric. 2003. p. 300.

13. L, Dunn. pneumonia classification, diagnosis and nursing mangement . 2005.

14. S, Davidson. principle and practice of medicine. 21th Edition. 2010. p. 682.

15. Pneumonia Methods Working Group The Defnition of pneumonia, the assessment. Scott JA, Wonodi C, Moisi JC, et al. 2012.

16. Pneumonia research is to reduce the child motility in doveloping coutries. J. Anthony G. Scott, W. Abdullah Brooks,J.S. Malik Peiris,. Washinghton : s.n., 2018, p. 1.

17. Epidemiology and etiology of childhood pneumonia. Rudan I, Pinto CB, Biloglav Z, Mulholland K, Campbell H. 2008.

18. Prevalence of pneumonia and its associated factors among under-five children in East Africa a systematic review and meta-analysis. Azmeraw, Biruk Beletew . Melaku BimereW . Ayelign Mengesha . Mesfin Wudu and Molla. 2020, p. 4.

19. Statistics, World Health. PNEUMONIA. s.I. : 2021, 2019. p. 6.

20. Revised who classification and managementof childhood pneumoia at health facilities. organization, world health. s.I. : 2021, 2021, p. 9.

21. Pneumonia. Rani S. Gereige, MD, MPH,*Pablo Marcelo Laufer, MDt. 10 October 2013, 2013, infectious diseases, Vol. 34, p. 21.

22. Childhood pneumonia in developing countries. Abdulkarim, W B R Johnson and A A. 2 March 2013, 2013, African Journal of Respiratory Medicine, Vol. 8, p. 9. 
23. Acute Pneumonia and Its Complications. Chitra S. Mani. January, 2020, 2020, PART II: Clinical Syndromes and Cardinal Features of Infectious Diseases: Approach to Diagnosis and Initial Management, SECTION D: Lower Respiratory Tract Infections, Vol. 34, p. 17.

\section{Figures}

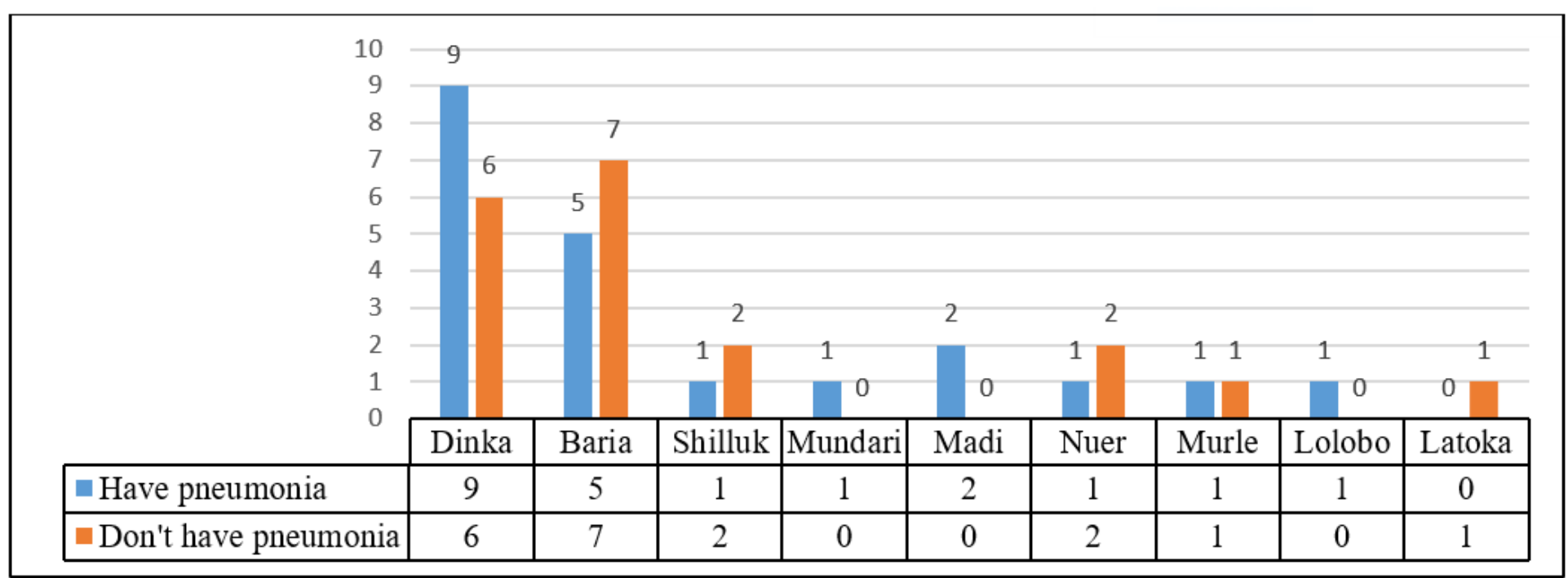

Figure 1

The Risk of Pneumonia by Ethnicity

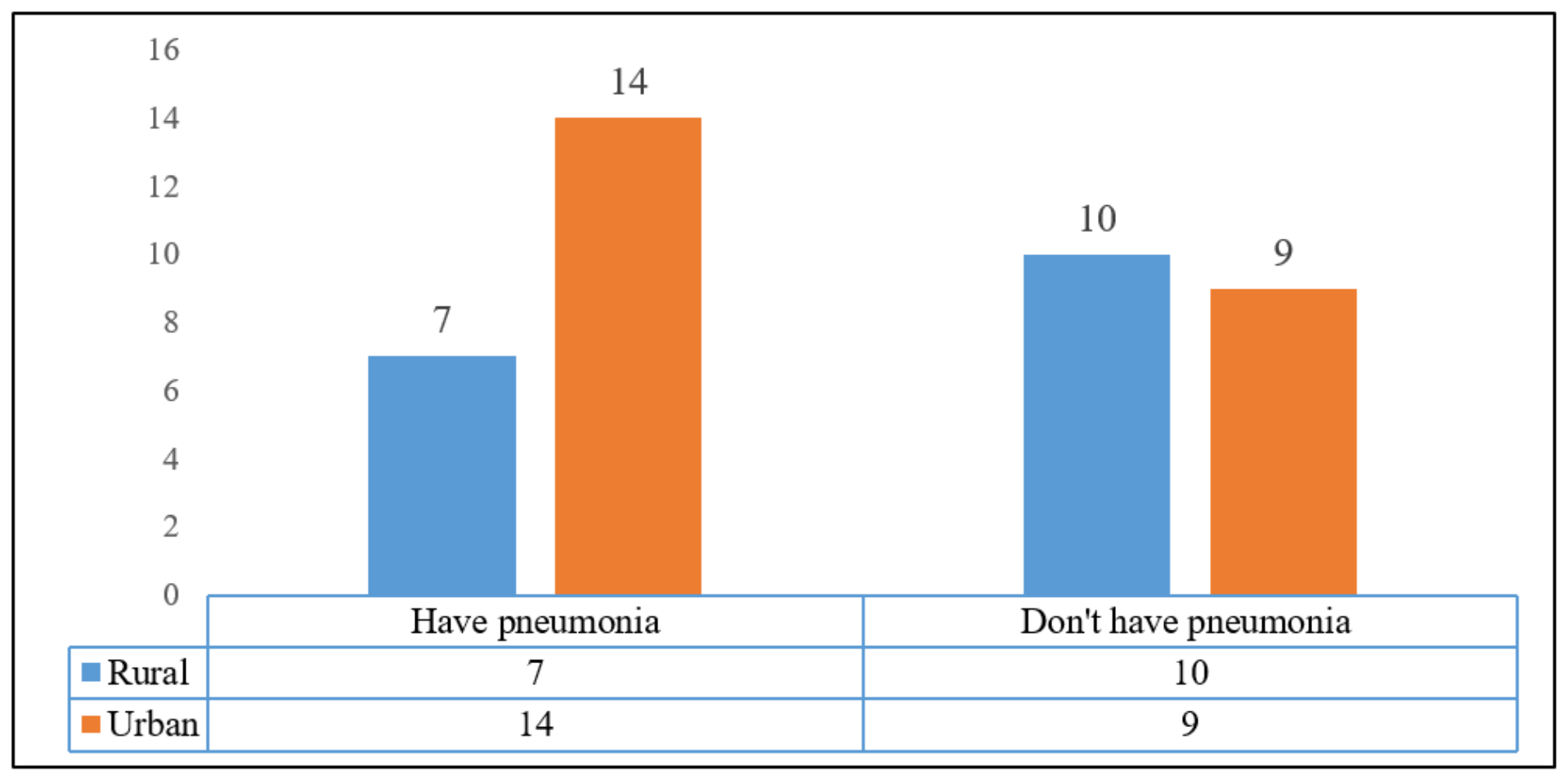

Figure 2 
the Risk of Pneumonia by Place of Residence

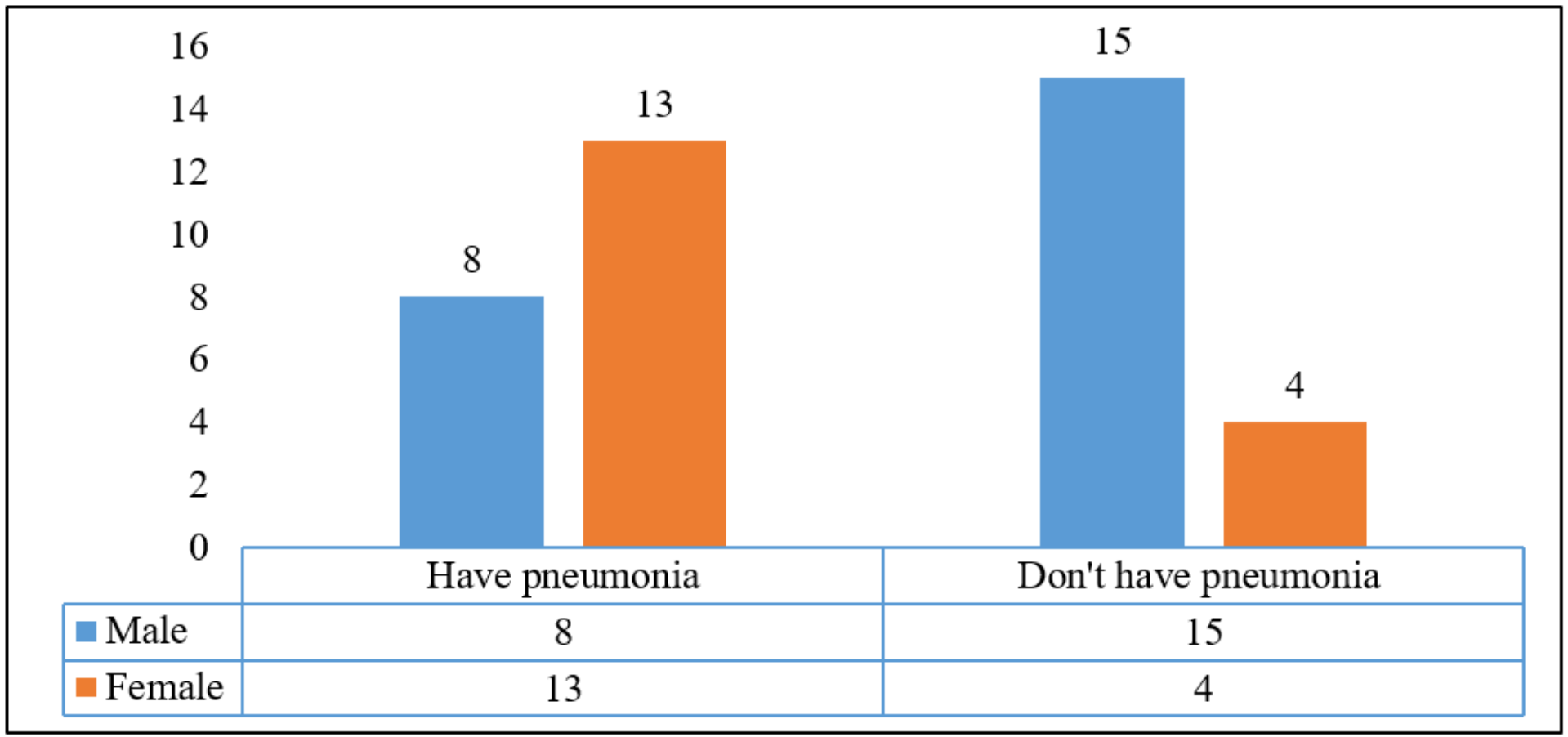

\section{Figure 3}

the Risk of Pneumonia by Sex of the child

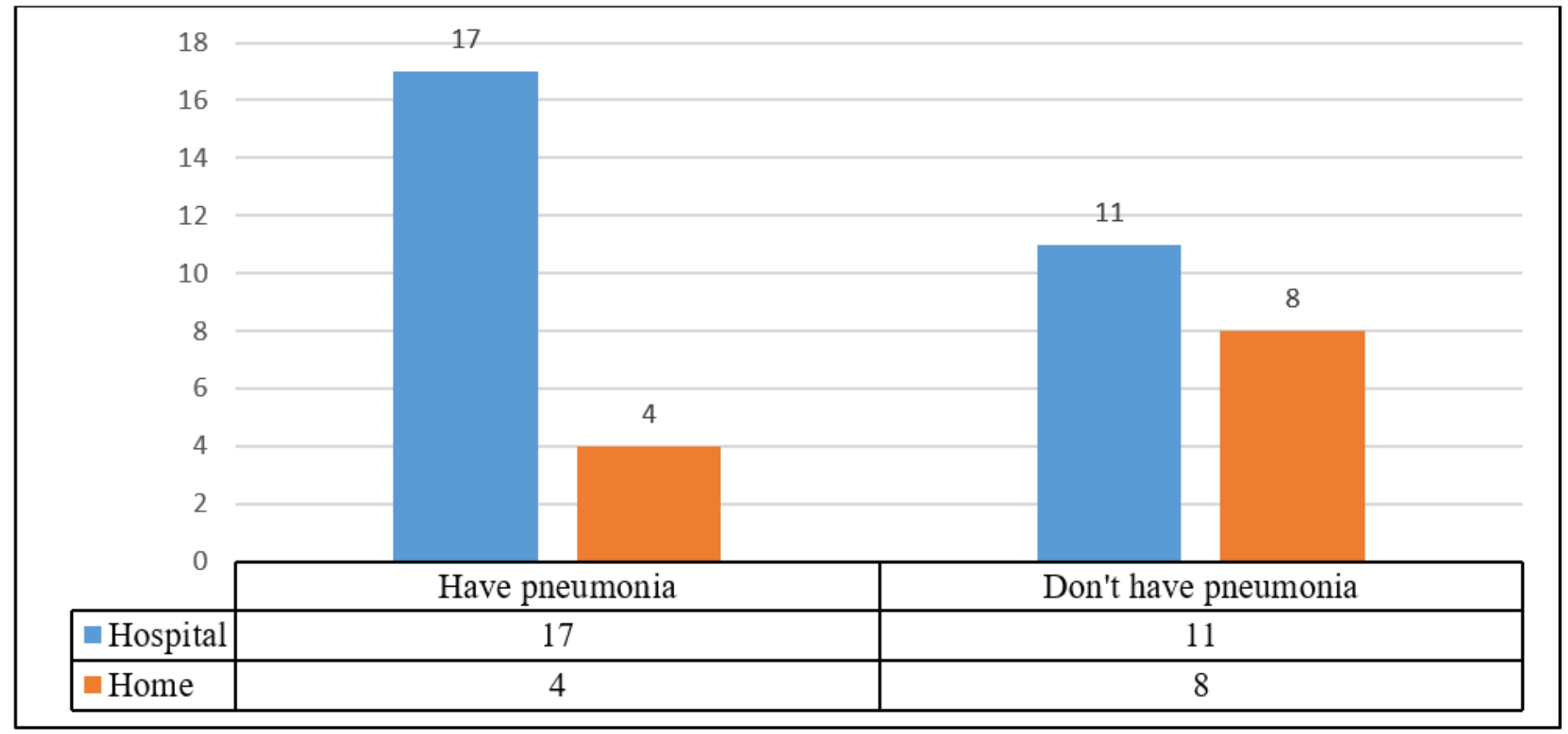

Figure 4

the Risk of Pneumonia by Place of Birth 
This is a list of supplementary files associated with this preprint. Click to download.

- Appendix.docx 